\title{
Silicon Improves Ion Homeostasis and Growth of Liquorice (Glycyrrhiza Uralensis Fisch. and Glycyrrhiza Inflata Bat.) Under Salt Stress by Reducing Plant $\mathrm{Na}^{+}$Uptake
}

\author{
Zihui Shen \\ Shihezi University \\ Xiaozhen Pu ( $\nabla$ xzh86936@163.com ) \\ Shihezi University \\ Shaoming Wang \\ Shihezi University \\ Xiuxiu Dong \\ Shihezi University \\ Xiaojiao Cheng \\ Shihezi University \\ Moxiang Cheng \\ Shihezi University
}

\section{Research Article}

Keywords: Silicon, salt stress, ion balance, photosynthetic pigment, uralensis

Posted Date: October 13th, 2021

DOI: https://doi.org/10.21203/rs.3.rs-899487/v1

License: (c) (1) This work is licensed under a Creative Commons Attribution 4.0 International License. Read Full License 


\section{Abstract}

Silicon effectively alleviates the damage caused by salt stress in plants and can improve plant salt tolerance. However, the details of the mechanism by which silicon improves salt tolerance of liquorice are limited, and the effects of foliar application of silicon on different liquorice species under salt stress are not known. Here, the effects of foliar spray of silicon on the growth, physiological and biochemical characteristics, and ion balance of Glycyrrhiza uralensis Fisch. and Glycyrrhiza inflata Bat. were investigated. High salt stress resulted in the accumulation of a large amount of $\mathrm{Na}+$, decreased photosynthetic pigment content, perturbed ion homeostasis, and eventually inhibited the both liquorice species growth. These effects were more pronounced in $\mathrm{G}$. uralensis, as $\mathrm{G}$. inflata is more salt tolerant than G. uralensis. Foliar spraying of silicon effectively reduced the decomposition of photosynthetic pigments, improved gas exchange parameters, and promoted photosynthesis. It also effectively inhibited lipid peroxidation and electrolyte leakage and enhanced osmotic adjustment of plants. Further, silicon application increased the $\mathrm{K}+$ concentration, reduced $\mathrm{Na}+$ absorption, transport and accumulation in the plants. The protective effects of silicon were more pronounced in G. uralensis than those in G. inflata. In conclusion, silicon reduces $\mathrm{Na}+$ absorption, improves ion balance, and alleviates the negative effects of salt stress in the two liquorice species studied, but the effect is liquorice species-dependent. These findings may inform novel strategies for protecting liquorice plants against salt stress and also provide a theoretical basis for the evaluation of salt tolerance and the scientific cultivation of liquorice.

\section{Introduction}

Soil salinisation is the main factor restricting agricultural development worldwide ${ }^{1}$. It has been estimated that $20 \%$ of the total cultivated land and $33 \%$ of the irrigated agricultural land is affected by high salinity ${ }^{2}$. Ion toxicity and osmotic stress are the main manifestations of salt damage in plants ${ }^{3}$. Ion toxicity causes plant roots and shoots to accumulate many harmful ions, such as $\mathrm{Na}^{+}$, under salinity stress. When the ions enter the plant cell, they destroy the water and ion balance, damage the organelle structure, and inhibit plant growth, even leading to plant death ${ }^{4}$. According to some studies, ion toxicity caused by $\mathrm{Na}^{+}$is more likely to cause irreversible damage to plant than osmotic stress ${ }^{5}$. Further, in plants, an antagonistic relationship exists between $\mathrm{Na}^{+}$and $\mathrm{K}^{+}$, whereby high concentrations of $\mathrm{Na}^{+}$directly interfere with and inhibit the absorption of $\mathrm{K}^{+}$by the plasma membrane ${ }^{6}$. The importance of $\mathrm{K}^{+}$in plant growth is second only to that of nitrogen, as the activation of more than 60 plant enzymes depends on $\mathrm{K}^{+7}$. Therefore, maintaining a high $\mathrm{K}^{+} / \mathrm{Na}^{+}$ratio under salt stress is essential for improving the salt tolerance of plants ${ }^{1}$.

Osmotic stress produces high levels of reactive oxygen species (ROS) under salt stress. Excessive ROS destroy macromolecules, lead to lipid peroxidation, eventually inhibit plant growth ${ }^{8}$. ROS clearance is mainly through enzymes and non-enzymatic antioxidant defence systems. Enzymes mainly include superoxide dismutase (SOD) and catalase (CAT), while non-enzymatic antioxidants mainly include the accumulation of compatible penetrants such as proline, soluble sugar, and soluble protein ${ }^{9}$. 
Silicon, which is usually absorbed by higher plants in the form of $\mathrm{H}_{4} \mathrm{SiO}_{4}{ }^{10}$, is the second most abundant element in the Earth's crust after oxygen ${ }^{11}$. While it is considered a non-essential element for the growth and development of higher plants ${ }^{12}$, many studies have shown that silicon plays an important role in alleviating biotic and abiotic stresses in plants ${ }^{13-16}$. Compared with other silicon application methods, foliar spray is the most effective ${ }^{17-18}$. Importantly, excessive application of silicon does not cause environmental pollution ${ }^{10}$.

Silicon accumulates in the cell wall of plants in the form of $\mathrm{SiO}_{2}$, which not only enhances the mechanical function of the cell wall, but also acts as a physical barrier to reduce water loss and improve the plant's ability to resist adverse environmental conditions ${ }^{19-20}$. Silicon also regulates polyamine metabolism, enhances $\mathrm{H}^{+}$-ATPase activity in the plasma membrane and the tonoplast, promotes $\mathrm{Na}^{+}$ excretion from the cell, and reduces $\mathrm{Na}^{+}$translocation and damage to plants ${ }^{21-22}$. Further, this element improves the activity of antioxidant enzymes (such as SOD and CAT), reduces electrolyte leakage (EL) ${ }^{23-}$ 24 , improves cell membrane stability, increases the water content of plant tissues, and improves photosynthetic function to promote plant growth ${ }^{25}$. Coskun, et al. ${ }^{26}$ recently proposed the 'apoplastic obstruction hypothesis' model, which explains the mechanism of silicon-mediated plant resistance to biotic and abiotic stresses.

Glycyrrhiza uralensis Fisch. and Glycyrrhiza inflata Bat. are the basic liquorice plants listed in the Chinese Pharmacopoeia ${ }^{27}$. Their roots and rhizomes have various pharmacological activities, such as antitumour $^{28}$, anti-viral ${ }^{29}$ and anti-inflammatory ${ }^{30}$. Further, liquorice plants survive in extreme environments, such as those characterised by low temperature, drought, and high salinity and alkalinity. Accordingly, the soil of the liquorice habitat is usually mildly, moderately, or severely salinized. The total salt content in the soil suitable for $G$. uralensis growth is $0-0.7 \%$ and that suitable for $G$. inflata is $0-1.3 \%{ }^{31}$. Consequently, liquorice is an important plant resource for improving saline-alkali soil, and preventing wind and sand erosion in the arid and semi-arid regions of Northwest China ${ }^{32}$.

Xinjiang is a vast territory and the main producer of liquorice in China. However, $31.1 \%$ of the existing cultivated land therein is damaged by salinity, so that $18 \%$ of that land is severely salinized, and $33 \%$ shows medium-level salinisation, which is detrimental to the growth of liquorice ${ }^{31}$. According to recent studies, at salt concentration exceeding $100 \mathrm{mmol} \mathrm{L}^{-1}$, the germination rate, germination potential, and germination index of $G$. uralensis seeds significantly decrease with a prolonged germination time. The plant height, chlorophyll content, and antioxidant enzyme activity of the seedlings are significantly reduced under these conditions, eventually leading to low biomass and low effective component accumulation in G. uralensis ${ }^{32-33}$.

The ameliorating effect of silicon application on plants under salt stress has been reported for many commercial crops, such as rice ${ }^{34}$, wheat ${ }^{35}$, sunflower ${ }^{36}$, sorghum ${ }^{37}$ and maize ${ }^{38}$, but the mechanisms involved vary among plant species. Further, to date, the understanding of the mechanism by which silicon 
improves the salt tolerance of liquorice is limited, and it is not known whether the application of silicon differently affects different liquorice species. In the current study, we focused on $G$. uralensis and $G$. inflata in Xinjiang, and explored the effects of foliar spray of silicon on the growth, physiological and biochemical characteristics, and ion balance of liquorice under salt stress, to clarify the mechanisms whereby silicon alleviates salt stress in these two liquorice species. This study provides a theoretical basis for the evaluation of salt tolerance and the scientific cultivation of liquorice.

\section{Materials And Methods}

\section{Plant material and growth conditions.}

A pot experiment was performed on the experimental field of the College of Life Science (Shihezi University, China; $\left.86^{\circ} 06 \mathrm{E}, 44^{\circ} 30 \mathrm{~N}\right)$. This region experiences a temperate continental climate, with a mean annual rainfall of $166 \mathrm{~mm}$, a mean annual temperature of $25.6^{\circ} \mathrm{C}, 169$ frost-free days, and annual sunshine of 3769 h. G. uralensis seeds were collected from Wenquan County (Xinjiang, China; $80^{\circ} 57 \mathrm{E}$, $44^{\circ} 58 \mathrm{~N}$ ), and $\mathrm{G}$. inflata seeds were collected from Korla (Xinjiang, China; $86^{\circ} 12 \mathrm{E}, 41^{\circ} 69 \mathrm{~N}$ ). The composition of sandy soil was as follows: $\mathrm{pH} \mathrm{7.63 \text {;silica, } 3 0 \mathrm { mg } \mathrm { kg }}$; ; soluble salt, $2.9 \mathrm{~g} \mathrm{~kg}^{-1}$; total nitrogen, $0.246 \mathrm{~g} \mathrm{~kg}^{-1}$; total phosphorus, $0.105 \mathrm{~g} \mathrm{~kg}^{-1}$; total potassium, $5.988 \mathrm{~g} \mathrm{~kg}^{-1}$; available nitrogen, $42.549 \mathrm{mg} \mathrm{kg}^{-1}$; available phosphorus, $4.231 \mathrm{mg} \mathrm{kg}^{-1}$; available potassium, $82.509 \mathrm{mg} \mathrm{kg}^{-1}$; and soil organic matter, $5.826 \mathrm{~g} \mathrm{~kg}^{-1}$.

\section{Experimental design.}

The treatments were arranged in a completely randomised block design with three replicates. To explore the effects of leaf application of silicon on the two liquorice species under different salt concentrations, the following treatments were chosen for detailed analysis: (1) control (CK); (2) control + $3 \mathrm{mM}$ silicon (CK + Si); (3) $6 \mathrm{~g} \mathrm{~kg}^{-1} \mathrm{NaCl}(6 \mathrm{~S})$; (4) $6 \mathrm{~g} \mathrm{~kg}^{-1} \mathrm{NaCl}+3 \mathrm{mM}$ silicon (6S + Si); (5) $12 \mathrm{~g} \mathrm{~kg}^{-1} \mathrm{NaCl}$ (12S); and (6) $12 \mathrm{~g} \mathrm{~kg}^{-1} \mathrm{NaCl}+3 \mathrm{mM}$ silicon (12S $+\mathrm{Si}$ ). Silicon was applied as a foliar spray in the form of potassium silicate $\left(\mathrm{K}_{2} \mathrm{SiO}_{3}\right)$. To account for the effect of potassium ions, a corresponding amount of potassium chloride was added to the treatments without silicon.

Ten seeds of liquorice were sown per plastic pot $\left(23.5^{\prime} 16^{\prime} 18 \mathrm{~cm}^{3}\right)$ containing $5 \mathrm{~kg}$ of sandy soil. After the emergence of 2 or 3 true leaves ( $15 \mathrm{~d}$ after sowing), six liquorice seedlings of the same height and growth, robust and disease-free were retained in each pot and were watered every day. $\mathrm{NaCl}$ and $\mathrm{Si}$ treatments were conducted simultaneously 4 weeks after sowing. Except for the treatments, other management measures were consistent with local field management practices. After $100 \mathrm{~d}$ of treatment, the growth parameters and physiological and biochemical characteristics of each liquorice species were determined.

\section{Determination of growth parameters of liquorice plants.}


Plant height was measured using a tape measure, and stem thickness was measured using a Vernier calliper. The leaves, stems, and roots of liquorice were washed and placed in an oven at $105^{\circ} \mathrm{C}$ for 30 min, dried at $75^{\circ} \mathrm{C}$ for $46 \mathrm{~h}$, and weighed.

\section{Determination of gas exchange attributes.}

Net photosynthesis $\left(P_{n}\right)$, net transpiration rate $\left(T_{r}\right)$, stomatal conductance $\left(g_{s}\right)$, and intercellular $\mathrm{CO}_{2}$ concentration $\left(C_{i}\right)$ were recorded between 9.00 a.m., and 12.00 a.m. using a Li-6400 photosynthesis instrument (Li-COR, Lincoln, NE, USA). Three similarly sized healthy and fully expanded leaves from the top of the stem from plants under each treatment were analysed at a leaf temperature of $28{ }^{\circ} \mathrm{C}$, irradiance of $1200 \mathrm{mmol} \mathrm{m}^{-2} \mathrm{~s}^{-1}$, and $\mathrm{CO}_{2}$ concentration of $400 \mathrm{mmol} \mathrm{mol}^{-1}$.

\section{Determination of chlorophyll content.}

The veins of fresh leaves were removed, cut into pieces, and weighed. Then, $10 \mathrm{~mL}$ of $80 \%(\mathrm{v} / \mathrm{v})$ acetone was added to $0.5 \mathrm{~g}$ of the plant material and extracted in the dark until the leaves were colourless. Next, the supernatant was obtained after centrifugation at $4000 \mathrm{r} \mathrm{min}^{-1}$ for $10 \mathrm{~min}$. Then we added $4 \mathrm{~mL}$ of $80 \%$ acetone to $1 \mathrm{ml}$ of supernatant, and the absorbance values of the extracts was determined at 470 $\mathrm{nm}, 646 \mathrm{~nm}$, and $663 \mathrm{~nm}$ using a Shimadzu UV-1900 spectrophotometer (Kyoto, Japan). The contents of chlorophyll $a$, chlorophyll $b$, total chlorophyll, and carotenoids were calculated by Lichtenthaler and Wellburn ${ }^{39}$.

\section{Determination of antioxidant enzyme activities.}

Fresh leaves $(0.5 \mathrm{~g})$ were added to phosphate buffer solution $(\mathrm{pH} 7.0)$, ground into a homogenate, and centrifuged at $10,000 \mathrm{r} \mathrm{min}^{-1}$ for $10 \mathrm{~min}$. The supernatant was diluted to $25 \mathrm{~mL}$ using the same buffer solution. The samples were then stored in an ice-box for the determination of SOD and catalase CAT activities. All operations were performed at $0-4{ }^{\circ} \mathrm{C}$. SOD activity was determined using the nitrogen blue tetrazole method ${ }^{40}$, and CAT activity was determined using the colorimetric method ${ }^{41}$.

\section{Determination of soluble sugar, soluble protein, and proline contents.}

For soluble sugar determinations, fresh leaves $(0.2 \mathrm{~g})$ were ground into a homogenate in $6 \mathrm{~mL}$ of distilled water, and then incubated in a water bath at $100^{\circ} \mathrm{C}$ for $20 \mathrm{~min}$. After cooling, the samples were centrifuged at $3000 \mathrm{r} \mathrm{min}^{-1}$ for $10 \mathrm{~min}$. The extract $(1.0 \mathrm{~mL})$ was then mixed with $5 \mathrm{~mL}$ of anthrone reagent, and absorbance value at $620 \mathrm{~nm}$ was measured by a Shimadzu UV-1900 spectrophotometer (Kyoto, Japan). The soluble sugar content was then calculated using a standard curve of sucrose.

Soluble protein was determined using the Coomassie Brilliant Blue G-250 method ${ }^{42}$. Fresh leaves $(0.2 \mathrm{~g})$ were added to phosphate buffer solution ( $\mathrm{pH} 7.0$ ), ground into a homogenate, and centrifuged at 5,000 r $\mathrm{min}^{-1}$ for $10 \mathrm{~min}$. Coomassie Brilliant Blue G-250 reagent was added to $1 \mathrm{~mL}$ of the supernatant and the 
absorbance was read at $595 \mathrm{~nm}$. The protein content was calculated using a standard curve of bovine serum albumin.

For proline content determinations, fresh liquorice leaves $(0.5 \mathrm{~g})$ were placed in $5 \mathrm{~mL}$ of $3 \%$ sulfosalicylic acid solution, and centrifuged at $5000 \mathrm{r} \mathrm{min}^{-1}$ for $10 \mathrm{~min}$. The supernatant $(2 \mathrm{~mL})$ was added to $2 \mathrm{~mL}$ of glacial acetic acid and $2 \mathrm{~mL}$ of acidic ninhydrin reagent, and the mixture was heated in a boiling water bath for $30 \mathrm{~min}$. After cooling, $4 \mathrm{~mL}$ of toluene was added, the samples were shaken for $30 \mathrm{~s}$, and centrifuged at $5000 \mathrm{r} \mathrm{min}^{-1}$ for $10 \mathrm{~min}$. Using toluene as a blank control, sample absorbance was measured at $520 \mathrm{~nm}$ by a Shimadzu UV-1900 spectrophotometer (Kyoto, Japan), and the proline content was calculated using a standard curve $\mathrm{e}^{43}$.

\section{Determination of lipid peroxidation and EL.}

Lipid peroxidation was determined by measuring the concentration of malondialdehyde (MDA). Liquorice leaves $(0.5 \mathrm{~g})$ were homogenised in $5 \mathrm{~mL}$ of $0.1 \%(\mathrm{w} / \mathrm{v})$ trichloroacetic acid solution. After centrifugation at $10,000 \mathrm{r} \mathrm{min}^{-1}$ for $10 \mathrm{~min}$, the supernatant was mixed with $0.5 \%(\mathrm{w} / \mathrm{v})$ trichloroacetic acid solution, and incubated in a water bath at $100{ }^{\circ} \mathrm{C}$ for $2 \mathrm{~min}$. The samples were then centrifuged at $10,000 \mathrm{r} \mathrm{min}^{-1}$ for $10 \mathrm{~min}$. The absorbance of sample supernatant at $600 \mathrm{~nm}, 532 \mathrm{~nm}$, and $450 \mathrm{~nm}$ was determined by a Shimadzu UV-1900 spectrophotometer (Kyoto, Japan), and the MDA content was calculated as described by Heath and Packer ${ }^{44}$.

To determine EL, fresh liquorice leaves were cleaned, cut into 2-cm pieces, and placed in a test tube containing $10 \mathrm{~mL}$ of distilled water. The samples were shaken on an oscillating table at $25^{\circ} \mathrm{C}$ for $24 \mathrm{~h}$ to determine electrical conductivity $1\left(\mathrm{EC}_{1}\right)$. The test tube was then placed in a water bath at $100{ }^{\circ} \mathrm{C}$ for 30 min to determine electrical conductivity $2\left(\mathrm{EC}_{2}\right)^{45}$. EL was calculated using the following formula: $\mathrm{EL}(\%)$ $=\mathrm{EC}_{1} / \mathrm{EC}_{2}{ }^{\prime} 100$.

\section{Determination of $\mathrm{Na}^{+}$and $\mathrm{K}^{+}$concentrations, transfer, and absorption.}

Dry plant samples ( $0.1 \mathrm{~g}$; various portions, as specified) were digested in a mixture of nitric acid and perchloric acid (volume ratio 2:1). The leaf and root concentrations of $\mathrm{Na}^{+}$and $\mathrm{K}^{+}$were measured using a flame photometer (FP640, Shanghai Precision Scientific Instrument Co., Ltd., Shanghai, China). Sodium uptake at the liquorice root surface, and ion $\left(\mathrm{Na}^{+}\right.$and $\left.\mathrm{K}^{+}\right)$translocation from the root to the shoot were calculated using the methods described by Yan, et al. ${ }^{46}$ and Ali, et al. ${ }^{38}$, respectively.

\section{Statistical analysis.}

The data were analysed using SPSS 20.0 (SPSS Inc., Chicago, IL, USA). One-way analysis of variance (ANOVA) was used to test the effect of different treatments on each index in the same liquorice species $(P<0.05)$, and Duncan's multiple comparisons test was used to determine significant differences between different treatments of the same liquorice. When the variances were not uniform, the data were 
converted to logarithm or square-root values before analysis. All figures were prepared using Origin 9 (OriginLab, Northampton, MA, USA).

\section{Results}

\section{Plant growth.}

Salt stress significantly inhibited the growth of the two liquorice species. The inhibitory effect on plant growth was more pronounced in G. uralensis than that in G. inflate. In particular, under the $12 \mathrm{~S}$ treatment, the plant height, root dry weight, and shoot dry weight of $G$. uralensis decreased by $55 \%, 88 \%$, and $84 \%$, respectively, compared with the corresponding CK values; for $G$. inflata, these indicators decreased by $41 \%, 86 \%$, and $83 \%$, respectively, in the same comparison. In addition, under the $12 \mathrm{~S}$ treatment, the plant height, root dry weight, and shoot dry weight of $G$. inflata were $57 \%, 39 \%$, and $42 \%$ higher, respectively, than those of $G$. uralensis (Table 1). This inhibitory effect was alleviated by silicon (Table 1). Under the $12 S+S i$ treatment, the plant height, root dry weight, and shoot dry weight of $G$. uralensis increased by $74 \%, 81 \%$, and $74 \%$, respectively, compared with the corresponding $12 \mathrm{~S}$ values; for $G$. inflata, these indicators increased by $19 \%, 48 \%$, and $49 \%$, respectively, in the same comparison. Furthermore, the application of silicon with the CK treatment increased the growth indicators of the two liquorice species.

\section{Gas exchange attributes.}

The $P_{n}, g_{s}, T_{n}$ and $C_{i}$ parameters of liquorice plants were generally negatively affected (Fig. 1$)$. Under the $6 \mathrm{~S}$ treatment, the $P_{n}, g_{S}$, and $\mathrm{T}_{\mathrm{r}}$ of $G$. uralensis were reduced by $54 \%, 64 \%$, and $62 \%$, respectively, compared with the corresponding CK values;for $G$. inflata, the corresponding parameters were reduced by $26 \%, 22 \%$, and $21 \%$, respectively, in the same comparison. Under the $12 \mathrm{~S}$ treatment, the $P_{n}, g_{s}, T_{n}$ and $C_{i}$ of $G$. inflata were decreased by $70 \%, 76 \%, 77 \%$, and $20 \%$, respectively, compared with the corresponding CK values; for $G$. uralensis, the corresponding parameters were also significantly decreased, except for $C_{i}$ which was increased by $41 \%$ in the same comparison. In addition, the $P_{n}, g_{s}$ and $T_{r}$ of $G$. inflata were $148 \%, 277 \%$, and $267 \%$ higher than those of $G$. uralensis under the $6 S$ treatment, respectively. Nevertheless, compared with the $6 \mathrm{~S}$ values, the $6 \mathrm{~S}+\mathrm{Si}$ treatment increased the $P_{n}, g_{S}, T_{n}$ and $C_{i}$ of $G$. uralensis by $78 \%, 59 \%, 55 \%$, and $33 \%$, respectively; it increased the corresponding parameters of $G$. inflata by $29 \%, 15 \%, 15 \%$, and $15 \%$, respectively. By contrast, the application of silicon with the $12 \mathrm{~S}$ treatment reduced the $C_{i}$ of $G$. uralensis (16\%).

\section{Chlorophyll concentration.}

Compared with the CK values, $12 \mathrm{~S}$ treatment significantly reduced the chlorophyll content of the two liquorice species (Fig. 2). Under the 12S treatment, the chlorophyll $a$, chlorophyll $b$, total chlorophyll, and carotenoid contents of $G$. uralensis were reduced by $65 \%, 53 \%, 63 \%$, and $65 \%$, respectively, compared with the corresponding CK values; for $G$. inflata, the corresponding parameters were reduced by $51 \%, 53 \%$, $52 \%$, and $57 \%$, respectively, in the same comparison. Further, under the $12 S$ treatment, the chlorophyll $a$, 
chlorophyll $b$, total chlorophyll, and carotenoid contents of $G$. inflata were $44 \%, 22 \%, 40 \%$, and $24 \%$ higher than those of $G$. uralensis, respectively. The application of silicon with the $12 S$ treatment resulted in an increase in the chlorophyll $a$ ( $49 \%$ and $30 \%$ ), chlorophyll $b$ (33\% and $5 \%$ ), total chlorophyll ( $46 \%$ and $26 \%$ ), and carotenoid (66\% and $50 \%$ ) contents of G. uralensis and G. inflata, accordingly. Further, the application of silicon with the CK treatment also increased the chlorophyll content of the two liquorice species.

\section{Antioxidant enzyme activities.}

With the increasing salt concentration, the activities of SOD and CAT in G. inflata were significantly increased, while those in $G$. uralensis increased initially and then decreased (Fig. 3). Compared with the corresponding CK values, the SOD and CAT activity in G. uralensis increased by $28 \%$ and $128 \%$, respectively, under the $6 \mathrm{~S}$ treatment; the corresponding parameters of $G$. inflata increased by $9 \%$ and $62 \%$, respectively, in the same comparison. Compared with the $6 \mathrm{~S}$ values, the SOD and CAT activities in $G$. inflata increased by $14 \%$ and $64 \%$, respectively, under the $12 \mathrm{~S}$ treatment; the corresponding parameters in G. uralensis decreased by $12 \%$ and $22 \%$, respectively, in the same comparison. In addition, under the $12 S$ treatment, the SOD and CAT activities in G. inflata were $20 \%$ and $69 \%$ higher than those of G. uralensis, respectively. Compared with the salt treatment, the application of silicon significantly increased the SOD and CAT activities in the two liquorice species $(P<0.05)$.

\section{Soluble sugar, soluble protein, and proline contents.}

The soluble sugar, soluble protein, and proline contents in the leaves of $G$. uralensis first increased and then decreased with an increasing salt concentration (Fig. 4). Compared with the corresponding CK values, the $6 S$ treatment increased the soluble sugar content of $G$. uralensis and $G$. inflata by $101 \%$ and $61 \%$, soluble protein content by $97 \%$ and $38 \%$, and proline content by $107 \%$ and $99 \%$, respectively. However, under the $12 S$ treatment, the soluble sugar, soluble protein, and proline contents of $G$. uralensis decreased by $30 \%, 17 \%$, and $20 \%$, respectively, compared with the corresponding $6 S$ values. In addition, under the $12 \mathrm{~S}$ treatment, the soluble protein and proline contents of $G$. inflata were $47 \%$ and $29 \%$ higher than those of $G$. uralensis, respectively. Compared with the absence of silicon, application of silicon significantly reduced the proline content of the two liquorice species $(P<0.05)$, and increased the soluble sugar and soluble protein contents. Further, the application of silicon with the $12 \mathrm{~S}$ treatment resulted in a $19 \%$ decrease of the proline content of G. uralensis, and a $53 \%$ and $20 \%$ increase in the soluble sugar and soluble protein contents, respectively, in this species.

\section{Lipid peroxidation and EL.}

Salt treatment significantly increased the MDA and EL of the two liquorice species $(P<0.05$; Fig. 5$)$. Under the $6 S$ and $12 S$ treatments, the MDA of $G$. uralensis increased by $82 \%$ and $161 \%$, respectively, and EL increased by $49 \%$ and $141 \%$, respectively, compared with the corresponding CK values. For the same conditions, the MDA of $G$. inflata increased by $52 \%$ and $118 \%$, respectively, and EL increased by $25 \%$ and $68 \%$, respectively, compared with the corresponding CK values. Further, under the $12 \mathrm{~S}$ treatment, the MDA 
and EL of G. uralensis were $36 \%$ and $48 \%$ higher than those of G. inflata, respectively. Furthermore, the application of silicon with the $12 \mathrm{~S}$ treatment resulted in the reduction of the MDA of G. uralensis by $33 \%$ and that of EL by $16 \%$; for $G$. inflata, the MDA was reduced by $19 \%$ and EL was reduced by $22 \%$.

\section{Accumulation of $\mathrm{Na}^{+}$and $\mathrm{K}^{+}$in the root and leaf.}

The accumulation of $\mathrm{Na}^{+}$in the root and leaf of the two liquorice species increased significantly with an increasing salt concentration, with a simultaneous significant decrease in the accumulation of $\mathrm{K}^{+}$(Fig. 6). Under the $12 \mathrm{~S}$ treatment, $\mathrm{Na}^{+}$levels in the root and leaf of G. uralensis increased by $109 \%$ and $265 \%$, respectively, while $\mathrm{K}^{+}$levels decreased by $31 \%$ and $58 \%$, respectively, compared with the corresponding CK values. Under the same conditions, $\mathrm{Na}^{+}$levels in $\mathrm{G}$. inflata increased by $181 \%$ and $246 \%$, accordingly, and $\mathrm{K}^{+}$levels decreased by $37 \%$ and $37 \%$, accordingly, compared with the corresponding $\mathrm{CK}$ values. In addition, under the $6 \mathrm{~S}$ treatment, the $\mathrm{Na}^{+}$and $\mathrm{K}^{+}$levels in the root, and $\mathrm{K}^{+}$levels in the leaf of $\mathrm{G}$. inflata were $44 \%, 27 \%$, and $50 \%$ higher, respectively, than those in G. uralensis. However, without silicon application (the $\mathrm{CK}, 6 \mathrm{~S}$, and $12 \mathrm{~S}$ treatments), the $\mathrm{Na}^{+}$accumulation in the leaf of $\mathrm{G}$. uralensis was $49 \%$, $35 \%$, and $57 \%$ higher than that in $G$. inflata, respectively. The application of silicon with the $6 \mathrm{~S}$ treatment significantly reduced $\mathrm{Na}^{+}$accumulation in the leaf of $\mathrm{G}$. uralensis $(27 \%)$, and increased $\mathrm{K}^{+}$accumulation in the leaf of this species (28\%).

\section{Ion translocation and uptake.}

Salt treatment significantly increased $\mathrm{Na}^{+}$transport (root-leaf) and $\mathrm{Na}^{+}$uptake on the root surface and decreased the $\mathrm{K}^{+} / \mathrm{Na}^{+}$ratio in both liquorice species (Fig. 7). Under the $6 \mathrm{~S}$ and $12 \mathrm{~S}$ treatments, compared with the corresponding $\mathrm{CK}$ values, $\mathrm{Na}^{+}$transport in G. uralensis increased by $56 \%$ and $73 \%$, respectively; $\mathrm{Na}^{+}$uptake on the root surface increased by 1.81 - and 22 -fold, respectively; $\mathrm{K}^{+}$transport (root-leaf) decreased by $30 \%$ and $40 \%$, respectively; and the $\mathrm{K}^{+} / \mathrm{Na}^{+}$ratio decreased by $59 \%$ and $81 \%$, respectively. In addition, the $\mathrm{K}^{+}$transport and $\mathrm{K}^{+} / \mathrm{Na}^{+}$ratio in $G$. inflata were $18 \%$ and $72 \%$ higher than those of $G$. uralensis under the $6 \mathrm{~S}$ treatment, respectively, while the $\mathrm{Na}^{+}$transport and $\mathrm{Na}^{+}$uptake on the root surface in G. uralensis were $95 \%$ and $85 \%$ higher than those of $G$. inflata, respectively. While the application of silicon decreased the $\mathrm{Na}^{+}$transport, and increased the $\mathrm{K}^{+}$transport and $\mathrm{K}^{+} / \mathrm{Na}^{+}$ratio, it only significantly affected the $\mathrm{Na}^{+}$uptake on the root surface under high salt concentration, decreasing the $\mathrm{Na}^{+}$uptake by $52 \%$ in G. uralensis and $46 \%$ in G. inflata.

\section{Discussion}

Salt stress is one of the main adverse environmental conditions faced by plants ${ }^{1}$. It inhibits plant growth and reduces plant yields, mainly because of ion toxicity and osmotic stress, and even leads to plant death ${ }^{47}$. According to many studies, silicon can be used to effectively alleviate the harmful effects of salt stress on plants and promote plant growth in saline environments ${ }^{48-49}$. In the current study, we analysed the growth indicators, gas exchange parameters, chlorophyll content, osmotic regulators, membrane 
damage indicators, antioxidant enzyme activity, and ion balance in two liquorice species. We showed that silicon application alleviated the negative effects of salt stress to a certain extent with the extent of the mitigating effect depending on the liquorice species. Although some investigations on the effect of silicon on the growth of liquorice under salt stress have been reported ${ }^{50-52}$, this is the first report demonstrating that foliar spray with silicon enhances the salt tolerance of $G$. uralensis and $G$. inflata.

Both the medium-salt (6S) and high-salt (12S) treatments significantly reduced the biomass of the two liquorice species, with the lowest values obtained at high salt concentrations (Table 1). Under the same salt treatment, the growth of $G$. inflata was better than that of $G$. uralensis, indicating that $G$. inflata is more salt tolerant than $G$. uralensis. The increase in $\mathrm{Na}^{+}$under salt treatment hindered the photosynthesis by reducing photosynthetic pigment levels and further reducing the photosynthetic rate ${ }^{17}$, thereby inhibiting plant growth and reducing the biomass (Table 1). Consistent with the findings for mung bean ${ }^{8}$ and cucumber ${ }^{53}$, salt stress reduced chlorophyll concentration and carotenoid levels in the leaves of the two liquorice species (Fig. 2). In addition, under high salt stress, the $P_{n}, T_{n}$ and $g_{s}$ of the two liquorice species were also significantly reduced. However, the $C_{\text {, of }} G$. uralensis was significantly increased under high salt stress, indicating that when the salt concentration exceeds the tolerance limit of a plant, the stomatal protection mechanism is damaged, and non-stomatal factors play a major role in photosynthesis ${ }^{54}$. Further, foliar spray with silicon significantly increased chlorophyll and carotenoid levels in plant leaves under salt stress ${ }^{17,55}$. These observations suggest that silicon promotes the biosynthesis of photosynthetic pigments under salt stress. This may be attributed to the notion that silicon alleviates the damage to chloroplast under salt stress ${ }^{51}$, enhances RuBisCo protein expression, and promotes the synthesis of chlorophyll and carotenoids ${ }^{48}$. Similarly, foliar spraying with silicon alleviated the negative effects of salt stress on gas exchange parameters in the two liquorice species (Fig. 1). The promoting effect was different for the different liquorice species. In the current study, the effect of silicon was more pronounced for G. uralensis (Fig. 2).

Under salt stress, the damage to plant cell and the decrease in photosynthesis are mainly caused by excessive absorption and accumulation of $\mathrm{Na}^{+17,35}$. Under high salt stress, the ability of the root system of $\mathrm{G}$. uralensis to intercept $\mathrm{Na}^{+}$was significantly decreased, and the accumulation of $\mathrm{Na}^{+}$in the root and leaf increased, which led to disturbance in the synthesis of photosynthetic pigments ${ }^{56}$, a decrease in the net photosynthetic rate, and a significant reduction of plant biomass (Figs 1a, 2, and Table 1). This indicates that the ability of the root to intercept $\mathrm{Na}^{+}$was limited under these conditions. In high-salt environments, the accumulation of $\mathrm{Na}^{+}$was increased in roots and the transport ability of $\mathrm{Na}^{+}$was enhanced toward the shoot, thus increasing $\mathrm{Na}^{+}$accumulation in the leaf and damaging mesophyll cells ${ }^{57}$. When the $\mathrm{Na}^{+}$concentration in the leaf exceeds $1.3 \mathrm{mg} \mathrm{g}^{-1}$, the chloroplast structure is destroyed, chlorophyll degradation accelerates, and photosynthesis is inhibited ${ }^{14}$. In addition, the transportation and absorption of $\mathrm{Na}^{+}$on the root surface of $G$. uralensis were 1.95 and 1.84 times higher than those in $G$. inflata under medium-salinity conditions. This indicates that the ability of $G$. inflata roots to intercept $\mathrm{Na}^{+}$ is greater than that of $G$. uralensis, which might account for the relatively high salt tolerance of $G$. inflata. 
$\mathrm{K}^{+}$is the key regulator of cell homeostasis ${ }^{7}$ and plays an important role in inducing cell elongation, maintaining osmotic regulation in plants, and promoting photosynthesis ${ }^{58-59}$. Therefore, excessive $\mathrm{Na}^{+}$ levels often lead to $\mathrm{K}^{+}$deficiency. Al-Huqail, et al. ${ }^{48}$ reported that high salt concentrations significantly increase the $\mathrm{Na}^{+}$content in maize and greatly reduce the $\mathrm{K}^{+}$content, resulting in an increased $\mathrm{Na}^{+} / \mathrm{K}^{+}$ ratio and plant growth inhibition. In G. uralensis, compared with the application of silicon in the soil, foliar spray alone effectively increased $\mathrm{K}^{+}$levels and reduced $\mathrm{Na}^{+}$levels ${ }^{50}$. This may be associated with the upregulation of genes involved in potassium uptake (OSAKT1 and OSSHAK1) and xylem load (OSSKOR) by silicon ${ }^{34}$, which promote the increase of $\mathrm{K}^{+}$transport and increase the $\mathrm{H}^{+}$-ATPase activity, forming a mechanical barrier to reduce $\mathrm{Na}^{+}$transport ${ }^{60}$. Therefore, we believe that the capacity of silicon to enhance $\mathrm{K}^{+}$-selective transport and increase the $\mathrm{K}^{+} / \mathrm{Na}^{+}$ratio might be one of the main mechanisms to improve plant growth and productivity under salt stress, which is in agreement with the findings of previous reports ${ }^{61}$. In addition, silicon not only reduced the $\mathrm{Na}^{+}$transfer and damage to the shoot under salt stress, but also decreased the $\mathrm{Na}^{+}$absorption by the root in the two liquorice species, which is consistent with the previous findings in rice ${ }^{62}$ and chickpea ${ }^{63}$. There are at least two possible explanations: (1) silicon deposition on the root cell wall enhances the mechanical strength of the cell wall, thus reducing $\mathrm{Na}^{+}$ absorption ${ }^{11}$; and (2) silicon decreases $\mathrm{Na}^{+}$accumulation in the root apex and cortex by upregulating the expression of ZmSOS1 and ZmSOS2 transporters ${ }^{64}$. Taken together, the reduction of $\mathrm{Na}^{+}$uptake and transport may be a potential mechanism of silicon-mediated enhancement of salt tolerance of liquorice.

In high-salt environments, plants accumulate high levels of $\mathrm{Na}^{+}$. This leads to excessive accumulation of ROS, perturbing the balance between their production and elimination, and resulting in cellular oxidative damage ${ }^{8}$. MDA and EL are the main indexes used to evaluate the severity of oxidative cell damage ${ }^{62}$. MDA is an oxidation product of membrane lipids and accumulates when plants are subjected to oxidative stress. $\mathrm{Na}^{+}$accumulation results in the production of high levels of ROS, which destroy the cell membrane structure, leading to an increased MDA content and EL. For instance, salt-induced oxidative damage leads to the rupturing of the plasma membrane in maize, leading to lipid peroxidation $^{38}$. We here showed that both medium and high-salt treatments increased the MDA content and $E L$ in the two liquorice species, especially in G. uralensis (Fig. 5), indicating major damage of the cell membrane in these liquorice species. Of the two species, the damage was more pronounced in $G$. uralensis. However, the MDA content and EL in the two liquorice species decreased after foliar spraying with silicon. These observations suggest that silicon may counteract the membrane damage caused by salt stress in liquorice. In addition, silicon can alleviate reactive oxygen damage by maintaining membrane integrity and activating the antioxidant defence system ${ }^{65}$. In the current study, silicon enhanced SOD and CAT activities in the two liquorice species under salt stress (Fig. 3), which was consistent with the observations in mung bean ${ }^{8}$ and mustard ${ }^{66}$. This effect may be related to the upregulation of genes encoding antioxidant enzymes ${ }^{67}$. We also observed that silicon application enhanced the SOD and CAT activities in $G$. uralensis to a greater extent than those in $G$. inflata. Therefore, 
the regulation of the plant antioxidant system by silicon upon salt stress is different for the two different liquorice species.

The alleviating effect of silicon described above was not only associated with the increased antioxidant enzyme activity, but also with the accumulation of osmotic regulators in plants ${ }^{48}$. Accordingly, upon salt stress, plants produce and accumulate compatible organic solutes as a means of osmotic adjustment, to maintain the normal physiological and biochemical characteristics of intracellular water, and to impede the damage to the cell membrane ${ }^{54,68}$. By contrast, under high-salt stress, the soluble sugar, soluble protein, and proline contents in the leaf of $G$. uralensis were decreased, indicating that high-salt stress greatly affected the ability of $G$. uralensis to respond to the adverse conditions, and inhibited plant growth $^{50}$. Proline is a key osmoprotectant and can reduce the damage caused by ROS, reduce lipid peroxidation, and protect protein and membrane structures ${ }^{54}$. In the current study, the application of silicon reduced the proline content in liquorice, which was consistent with the findings in sunflower ${ }^{36}$ and pelargonium ${ }^{24}$. In the presence of silicon, proline was degraded, and used as a source of carbon and nitrogen in plants recovering from stress, as well as a membrane stabiliser and a free-radical scavenger, to reduce lipid peroxidation and $\mathrm{EL}^{15,18}$. Therefore, reduction of membrane damage may be another mechanism whereby silicon improves the salt tolerance of plants. However, the mechanism for obtaining silicon to improve plant salt tolerance is a complex process. In addition to many physiological and biochemical aspects, further research is required on protein and gene expression analysis.

\section{Conclusion}

High salinity greatly inhibited the growth and development of the two liquorice species studied herein. Under salt stress, the morphological characteristics (plant height, stem diameter, and biomass), photosynthetic characteristics (gas exchange parameters and photosynthetic pigments), antioxidant enzyme activities (SOD and CAT), ion homeostasis ( $\mathrm{K}^{\square}$ and $\mathrm{Na}^{\square}$ transport, and $\mathrm{K}^{\square} / \mathrm{Na}^{\square}$ ratio), and osmotic adjustment (e.g. proline) in $G$. inflata were better than those in $G$. uralensis. These observations indicate that $G$. inflata is more salt tolerant than $G$. uralensis. Foliar application of silicon effectively reduced the absorption of $\mathrm{Na}^{+}$, improved ion balance, alleviated membrane damage, and promoted the growth of the two liquorice species. Further, the response of $G$. uralensis to silicon was more pronounced than that of $G$. inflata, indicating that the protective effect of silicon is different for different liquorice species. This study provides a theoretical basis for the evaluation of salt tolerance in and scientific artificial cultivation of liquorice in the future.

\section{Declarations}

Acknowledgments This work was supported by the National Natural Science Foundation of China (31660361), and the Director fund of Education Key Laboratory of Xinjiang Phytomedicine Resource Utilization (XPRU202003). 
Conflict of interest All authors declared no conflict of interest.

Authors' contributions SZH and PXZ conceived the study and participated in its design. SZH, DXX, and CXJ contributed samples and carried out the experiments. SZH, CMX, and PXZ analysed the data. SZH, PXZ and WSM drafted the manuscript. The authors have approved of publication, and there is no conflict of interest. All the authors equally approve of publication.

Compliance statement for experimental materials Liquorice is a widely distributed species in China. Xinjiang is the main producing area of liquorice in China. Seed of $G$. uralensis was collected from Wenquan County (Xinjiang, China; $80^{\circ} 57 \mathrm{E}, 44^{\circ} 58 \mathrm{~N}$ ), and that of $G$. inflata was collected from Korla (Xinjiang, China; $86^{\circ} 12 \mathrm{E}, 41^{\circ} 69 \mathrm{~N}$ ). A pot experiment was performed on the experimental field of the College of Life Science (Shihezi University, China; $86^{\circ} 06 \mathrm{E}, 44^{\circ} 30 \mathrm{~N}$ ). Therefore, all operations comply with relevant institutional, national, and international guidelines and legislation.

\section{References}

1. Zhao, S. S., Zhang, Q. K., Liu, M. Y., Zhou, H. P. Ma, C. L. \& Wang, P. P. Regulation of plant responses to salt stress. Int. J. Mol. Sci. 22, 16 (2021).

2. Shrivastava, P. \& Kumar, R. Soil salinity: a serious environmental issue and plant growth promoting bacteria as one of the tools for its alleviation. Saudi J. Biol. Sci. 22, 123-131 (2015).

3. Ramon Acosta-Motos, J. et al. Plant responses to salt stress: adaptive mechanisms. AgronomyBasel. 7, (2017).

4. Munns, R. \& Tester, M. Mechanisms of salinity tolerance. Annu. Rev. Plant Biol. 59, 651-681 (2008).

5. Wu, H. H. Plant salt tolerance and $\mathrm{Na}^{+}$sensing and transport. Crop Journal. 6, 215-225 (2018).

6. Zelm, E. V., Zhang, Y. X. \& Testerink, C. Salt tolerance mechanisms of plants (ed. Merchant, S. S.) 403$433(2020)$.

7. Kumar, P., Kumar, T., Singh, S., Tuteja, N., Prasad, R. \& Singh, J. Potassium: a key modulator for cell homeostasis. J. Biotechnol. 324, 198-210 (2020).

8. Ahmad, P. et al. Silicon (si) supplementation alleviates nacl toxicity in mung bean vigna radiata (I.) wilczek through the modifications of physio-biochemical attributes and key antioxidant enzymes. $J$. Plant Growth Regul. 38, 70-82 (2019).

9. Chiappero, J. et al. Antioxidant status of medicinal and aromatic plants under the influence of growth-promoting rhizobacteria and osmotic stress. Industrial Crops and Products. 167, (2021).

10. Etesami, H. \& Jeong, B. R. Silicon (si): review and future prospects on the action mechanisms in alleviating biotic and abiotic stresses in plants. Ecotoxicol. Environ. Saf. 147, 881-896 (2018).

11. Epstein, E., Silicon. Annu. Rev. Plant Physiol. Plant Mol. Biol. 50, 641-664 (1999).

12. Epstein, E., The anomaly of silicon in plant biology. Proc. Natl. Acad. Sci. U. S. A. 91, 11-7 (1994).

13. Jadhao, K. R., Bansal, A. \& Rout, G. R. Silicon amendment induces synergistic plant defense mechanism against pink stem borer (Sesamia inferens Walker.) in finger millet (Eleusine coracana 
Gaertn.). Sci Rep. 10, 15 (2020).

14. Khan, W. U. D. et al. M. Silicon nutrition mitigates salinity stress in maize by modulating ion accumulation, photosynthesis, and antioxidants. Photosynthetica. 56, 1047-1057 (2018).

15. Li, Z. C. et al. Silicon enhancement of estimated plant biomass carbon accumulation under abiotic and biotic stresses. A meta-analysis. Agron. Sustain. Dev. 38, 19 (2018).

16. Cui, J. J., Zhang, E. H., Zhang, X. H. \& Wang, Q. Silicon alleviates salinity stress in licorice (Glycyrrhiza uralensis) by regulating carbon and nitrogen metabolism. Sci Rep 11, 12 (2021).

17. Farouk, S., Elhindi, K. M. \& Alotaibi, M. A. Silicon supplementation mitigates salinity stress on Ocimum basilicum L. via improving water balance, ion homeostasis, and antioxidant defense system. Ecotoxicol. Environ. Saf. 206, 11 (2020).

18. Hurtado, A. C. et al. Different methods of silicon application attenuate salt stress in sorghum and sunflower by modifying the antioxidative defense mechanism. Ecotoxicol. Environ. Saf. 203, 11 (2020).

19. Gaur, S. et al. Fascinating impact of silicon and silicon transporters in plants: A review. Ecotoxicol. Environ. Saf. 202, 12 (2020).

20. Vandegeer, R. K. et al. Silicon deposition on guard cells increases stomatal sensitivity as mediated by $\mathrm{K}(+)$ efflux and consequently reduces stomatal conductance. Physiol. Plant 171, 358-370 (2021).

21. Lina et al. Silicon-mediated changes in polyamines participate in silicon-induced salt tolerance in Sorghum bicolorL. Plant, Cell Environ. 39, 245-258 (2016).

22. Alsaeedi, A. H. et al. Engineered silica nanoparticles alleviate the detrimental effects of $\mathrm{Na}^{+}$stress on germination and growth of common bean (Phaseolus vulgaris). Environ. Sci. Pollut. Res. 24, 2191721928 (2017).

23. Zhang, Z. Z. et al. Alleviating effect of silicon on melon seed germination under autotoxicity stress. Ecotoxicol. Environ. Saf. 188, 9 (2020).

24. Hassanvand, F., Nejad, A. R. \& Fanourakis, D. Morphological and physiological components mediating the silicon-induced enhancement of geranium essential oil yield under saline conditions. Industrial Crops and Products. 134, 19-25 (2019).

25. Altuntas, O., Dasgan, H. Y. \& Akhoundnejad, Y. Silicon-induced Salinity Tolerance Improves Photosynthesis, Leaf Water Status, Membrane Stability, and Growth in Pepper (Capsicum annuum L.). HortScience. 53, 1820-1826 (2018).

26. Coskun, D. et al. The controversies of silicon's role in plant biology. New Phytol. 221, 67-85 (2019).

27. Jiang, M. Y. et al. An "essential herbal medicine"-licorice: A review of phytochemicals and its effects in combination preparations. J. Ethnopharmacol. 249, 14 (2020).

28. Zhang, X. Y. et al. Inhibition effect of glycyrrhiza polysaccharide (GCP) on tumor growth through regulation of the gut microbiota composition. J. Pharmacol. Sci. 137, 324-332 (2018).

29. Baltina, L. A. et al. Glycyrrhetinic acid derivatives as Zika virus inhibitors: Synthesis and antiviral activity in vitro. Bioorg. Med. Chem. 41, 116204 (2021). 
30. Zhao, Z. Y. et al. Glycyrrhizic acid nanoparticles as antiviral and anti-inflammatory agents for covid19 treatment. ACS Appl. Mater. Interfaces. 13, 20995-21006 (2021).

31. Lu, J. H., Lv, X., Wu, L. \& Li, X. Y. Germination responses of three medicinal licorices to saline environments and their suitable ecological regions. Acta Prataculturae Sinica. 22, 198-205 (2013).

32. Geng, G. Q. \& Xie, X. R. Effect of drought and salt stress on the physiological and biochemical characteristics of Glycyrrhiza uralensis. Pratacultural Science. 35, 113-120 (2018).

33. Cui, J. J., Zhang, X. H., Li, Y. T., Zhou, D. \& Zhang, E. H. Effect of silicon addition on seedling morphological and physiological indicators of Glycyrrhiza uralensis under salt stress. Acta Prataculturae Sinica. 24, 214-220 (2015).

34. Yan, G. C. et al. Silicon alleviates salt stress-induced potassium deficiency by promoting potassium uptake and translocation in rice (Oryza sativa L.). J. Plant Physiol. 258, 7 (2021).

35. Javaid, T., Farooq, M. A., Akhtar, J., Saqib, Z. A. \& Anwar-ul-Haq, M. Silicon nutrition improves growth of salt-stressed wheat by modulating flows and partitioning of $\mathrm{Na}^{+}, \mathrm{Cl}^{-}$and mineral ions. Plant Physiol. Biochem. 141, 291-299 (2019).

36. Conceicao, S. S. et al. Silicon modulates the activity of antioxidant enzymes and nitrogen compounds in sunflower plants under salt stress. Arch. Agron. Soil Sci. 65, 1237-1247 (2019).

37. Chen, D. Q. et al. Silicon moderated the K deficiency by improving the plant-water status in sorghum. Sci Rep 6, 14 (2016).

38. Ali, M. et al. Silicon mediated improvement in the growth and ion homeostasis by decreasing $\mathrm{Na}^{+}$ uptake in maize (Zea mays L.) cultivars exposed to salinity stress. Plant Physiol. Biochem. 158, 208218 (2021).

39. Lichtenthaler, H. K. \& Wellburn, A. R. Determinations of total carotenoids and chlorophylls a and b of leaf extracts in different solvents. Analysis 11, 591-592 (1983).

40. Beyer, W. F., Jr. \& Fridovich, I. Assaying for superoxide dismutase activity: some large consequences of minor changes in conditions. Anal. Biochem. 161, 559-66 (1987).

41. Cakmak, I. \& Marschner, H. Magnesium deficiency and high light intensity enhance activities of superoxide dismutase, ascorbate peroxidase, and glutathione reductase in bean leaves. Plant Physiol. 98, 1222-7 (1992).

42. Bradford, M. M. A rapid and sensitive method for the quantitation of microgram quantities of protein utilizing the principle of protein-dye binding. Anal. Biochem. 72, 248-54 (1976).

43. Bates, L. S., Waldren, R. P. \& Teare, I. D. Rapid Determination of Free Proline for Water-Stress Studies. Plant Soil. 39, 205-207 (1973).

44. Heath, R. L. \& Packer, L. Photoperoxidation in isolated chloroplasts. I. Kinetics and stoichiometry of fatty acid peroxidation. Arch. Biochem. Biophys. 125, 189-98 (1968).

45. Lutts, S., Kinet, J. M. \& Bouharmont, J. NaCl-induced senescence in leaves of rice (Oryza sativa L) cultivars differing in salinity resistance. Annals of Botany 78, 389-398 (1996). 
46. Yan, K., Wu, C. W., Zhang, L. H. \& Chen, X. B. Contrasting photosynthesis and photoinhibition in tetraploid and its autodiploid honeysuckle (Lonicera japonica Thunb.) under salt stress. Front. Plant Sci. 6, 9 (2015).

47. Rizwan, M. et al. Mechanisms of silicon-mediated alleviation of drought and salt stress in plants: a review. Environ. Sci. Pollut. Res. 22, 15416-15431 (2015).

48. Al-Huqail, A. A. et al. Silicon supplementation modulates antioxidant system and osmolyte accumulation to balance salt stress in Acacia gerrardii Benth. Saudi J. Biol. Sci. 26, 1856-1864 (2019).

49. Hurtado, A. C. et al. Silicon application induces changes C:N:P stoichiometry and enhances stoichiometric homeostasis of sorghum and sunflower plants under salt stress. Saudi J. Biol. Sci. 27, 3711-3719 (2020).

50. Zhang, W. J. et al. Silicon promotes growth and root yield of Glycyrrhiza uralensis under salt and drought stresses through enhancing osmotic adjustment and regulating antioxidant metabolism. Crop Prot. 107, 1-11 (2018).

51. Zhang, W. J. et al. Silicon alleviates salt and drought stress of Glycyrrhiza uralensis plants by improving photosynthesis and water status. Biol. Plant. 64, 302-313 (2020).

52. Zhang, X. H., Zhang, W. J., Lang, D. Y., Cui, J. J. \& Li, Y. T. Silicon improves salt tolerance of Glycyrrhiza uralensis Fisch by ameliorating osmotic and oxidative stresses and improving phytohormonal balance. Environ. Sci. Pollut. Res. 25, 25916-25932 (2018).

53. Yin, J. L. et al. Silicon enhances the salt tolerance of cucumber through increasing polyamine accumulation and decreasing oxidative damage. Ecotoxicol. Environ. Saf. 169, 8-17 (2019).

54. Liang, W. J., Ma, X. L., Wan, P. \& Liu, L. Y. Plant salt-tolerance mechanism: A review. Biochem. Biophys. Res. Commun. 495, 286-291 (2018).

55. Abdelaal, K. A. A., Mazrou, Y. S. A. \& Hafez, Y. M. Silicon foliar application mitigates salt stress in sweet pepper plants by enhancing water status, photosynthesis, antioxidant enzyme activity and fruit yield. Plants-Base/ 9, 1-15 (2020).

56. Trejo-Tellez, L. I. et al. Silicon induces hormetic dose-response effects on growth and concentrations of chlorophylls, amino acids and sugars in pepper plants during the early developmental stage. Peerj 8, 28 (2020).

57. Tester, M. \& Davenport, R., $\mathrm{Na}^{+}$tolerance and $\mathrm{Na}^{+}$transport in higher plants. Annals of Botany. 91, 503-527 (2003).

58. Zahoor, R. et al. Potassium fertilizer improves drought stress alleviation potential in cotton by enhancing photosynthesis and carbohydrate metabolism. Environ. Exp. Bot. 137, 73-83 (2017).

59. Khan, W. U. D., Aziz, T., Hussain, I., Ramzani, P. M. A. \& Reichenauer, T. G. Silicon: a beneficial nutrient for maize crop to enhance photochemical efficiency of photosystem II under salt stress. Arch. Agron. Soil Sci. 63, 599-611 (2017). 
60. Dhiman, P. et al. Fascinating role of silicon to combat salinity stress in plants: An updated overview. Plant Physiol. Biochem. 162, 110-123 (2021).

61. Hurtado, A. C. et al. Silicon alleviates sodium toxicity in sorghum and sunflower plants by enhancing ionic homeostasis in roots and shoots and increasing dry matter accumulation. Silicon. 13, 475-486 (2021).

62. Yan, G. C. et al. Silicon improves rice salinity resistance by alleviating ionic toxicity and osmotic constraint in an organ-specific pattern. Front. Plant Sci. 11, 12 (2020).

63. Garg, N. \& Bhandari, P. Silicon nutrition and mycorrhizal inoculations improve growth, nutrient status, $\mathrm{K}^{+} / \mathrm{Na}^{+}$ratio and yield of Cicer arietinum L. genotypes under salinity stress. Plant Growth Regul. 78, 371-387 (2016).

64. Bosnic, P., Bosnic, D., Jasnic, J. \& Nikolic, M. Silicon mediates sodium transport and partitioning in maize under moderate salt stress. Environ. Exp. Bot. 155, 681-687 (2018).

65. Das, P., Manna, I., Biswas, A. K. \& Bandyopadhyay, M. Exogenous silicon alters ascorbate-glutathione cycle in two salt-stressed indica rice cultivars (MTU 1010 and Nonabokra). Environ. Sci. Pollut. Res. 25, 26625-26642 (2018).

66. Alamri, S. et al. Silicon-induced postponement of leaf senescence is accompanied by modulation of antioxidative defense and ion homeostasis in mustard (Brassica juncea) seedlings exposed to salinity and drought stress. Plant Physiol. Biochem. 157, 47-59 (2020).

67. Ahmad, P. et al. Nitric oxide mitigates salt stress by regulating levels of osmolytes and antioxidant enzymes in chickpea. front. Plant Sci. 7, 1-11 (2016).

68. Zhu, Y. X. et al. Silicon confers cucumber resistance to salinity stress through regulation of proline and cytokinins. Plant Physiol. Biochem. 156, 209-220 (2020).

\section{Tables}

Table 1 Effects of silicon on the growth of Glycyrrhiza uralensis and G. inflata under salt stress 


\begin{tabular}{|c|c|c|c|c|c|}
\hline \multirow[t]{2}{*}{ Cultivar } & \multirow[t]{2}{*}{ Treatment } & Plant height & Stem diameter & Root DW & Shoot DW \\
\hline & & $(\mathrm{cm})$ & $(\mathrm{mm})$ & (g) & (g) \\
\hline \multirow[t]{6}{*}{ G. uralensis } & CK & $25.53 \pm 0.88 b$ & $2.06 \pm 0.11 b$ & $3.09 \pm 0.10 b$ & $2.34 \pm 0.09 a$ \\
\hline & $6 S$ & $18.6 \pm 0.72 c$ & $1.75 \pm 0.06 b c$ & $1.77 \pm 0.05 c$ & $1.67 \pm 0.03 b$ \\
\hline & $12 \mathrm{~S}$ & $11.43 \pm 0.06 d$ & $1.48 \pm 0.07 c$ & $0.36 \pm 0.01 d$ & $0.38 \pm 0.01 d$ \\
\hline & $\mathrm{CK}+\mathrm{Si}$ & $33.20 \pm 1.42 a$ & $2.95 \pm 0.18 a$ & $3.85 \pm 0.40 a$ & $2.51 \pm 0.11 \mathrm{a}$ \\
\hline & $6 S+S i$ & $26.30 \pm 0.06 b$ & $2.15 \pm 0.12 b$ & $2.16 \pm 0.08 c$ & $1.74 \pm 0.05 b$ \\
\hline & $12 S+S i$ & $19.87 \pm 0.34 c$ & $1.89 \pm 0.16 b$ & $0.66 \pm 0.02 d$ & $0.66 \pm 0.02 c$ \\
\hline \multirow[t]{6}{*}{ G. inflata } & CK & $30.47 \pm 0.78 b$ & $2.31 \pm 0.04 b$ & $3.54 \pm 0.04 b$ & $3.20 \pm 0.09 a$ \\
\hline & $6 S$ & $22.33 \pm 0.94 d$ & $1.97 \pm 0.02 c$ & $2.80 \pm 0.05 d$ & $2.26 \pm 0.03 b$ \\
\hline & $12 S$ & $18.00 \pm 1.26 d$ & $1.67 \pm 0.02 d$ & $0.51 \pm 0.02 f$ & $0.53 \pm 0.01 \mathrm{c}$ \\
\hline & $\mathrm{CK}+\mathrm{Si}$ & $38.97 \pm 0.47 a$ & $2.87 \pm 0.21 a$ & $4.24 \pm 0.07 a$ & $3.42 \pm 0.21 a$ \\
\hline & $6 S+S i$ & $26.83 \pm 1.19 c$ & $2.45 \pm 0.01 b$ & $3.40 \pm 0.02 c$ & $2.34 \pm 0.22 b$ \\
\hline & $12 S+S i$ & $21.33 \pm 0.93 d$ & $1.84 \pm 0.05 \mathrm{~cd}$ & $0.75 \pm 0.02 \mathrm{e}$ & $0.80 \pm 0.01 c$ \\
\hline
\end{tabular}

Data are presented as the mean $\pm S E(n=3)$. Different letters indicate significant differences between the values for the same index for the same liquorice species under different treatments $(P<0.05)$. DW, dry weight.

\section{Figures}



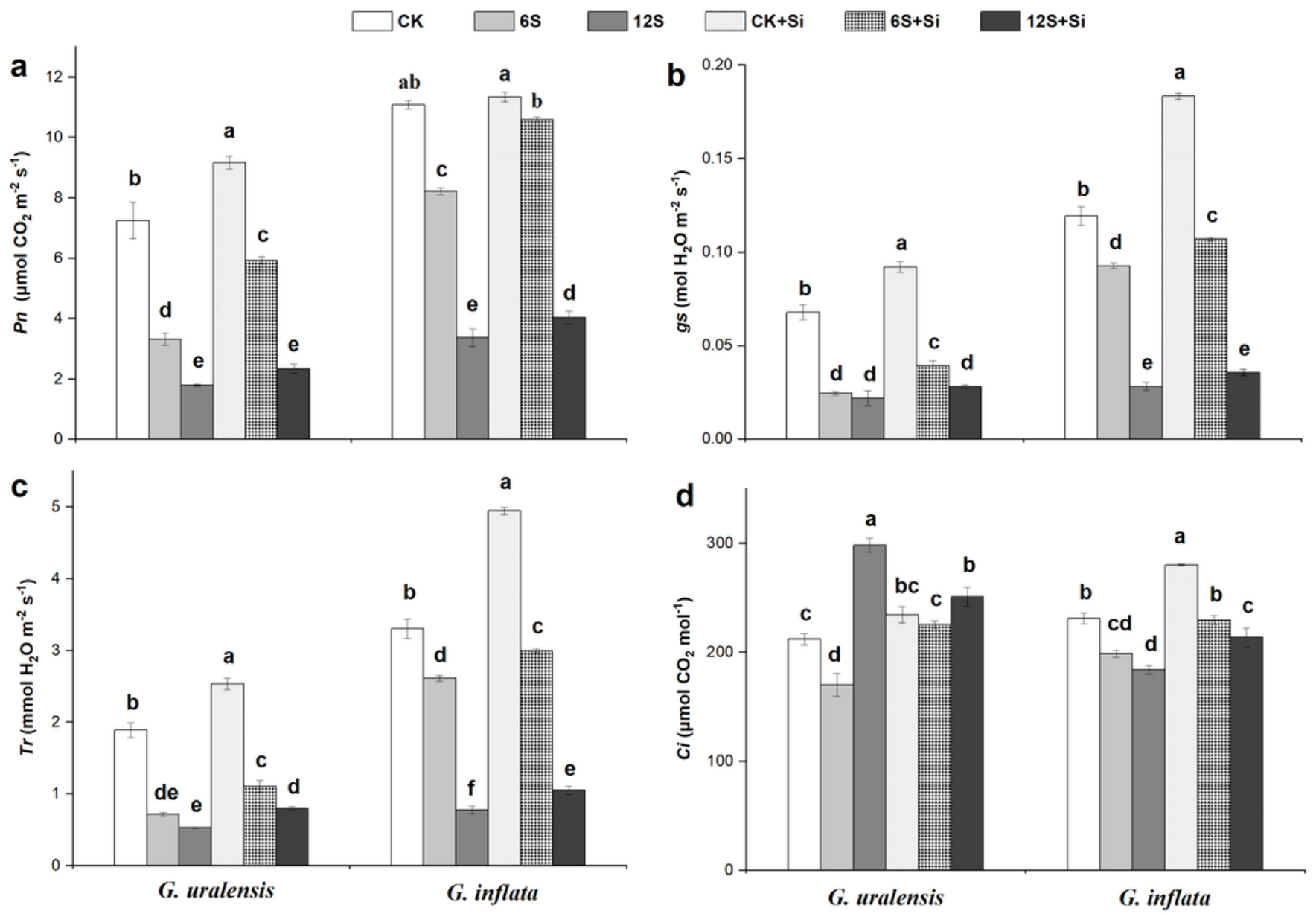

Figure 1

Effects of salinity and silicon on net photosynthetic rate (Pn, a), stomatal conductance (gs, b), transpiration rate $(\mathrm{Tr}, \mathrm{c})$, and intercellular $\mathrm{CO} 2$ concentration $(\mathrm{Ci}, \mathrm{d})$ in the two liquorice species. Data are presented as the mean $\pm S E(n=3)$. Different letters indicate significant differences between the values for one index for the same liquorice species under different treatments $(P<0.05)$ 

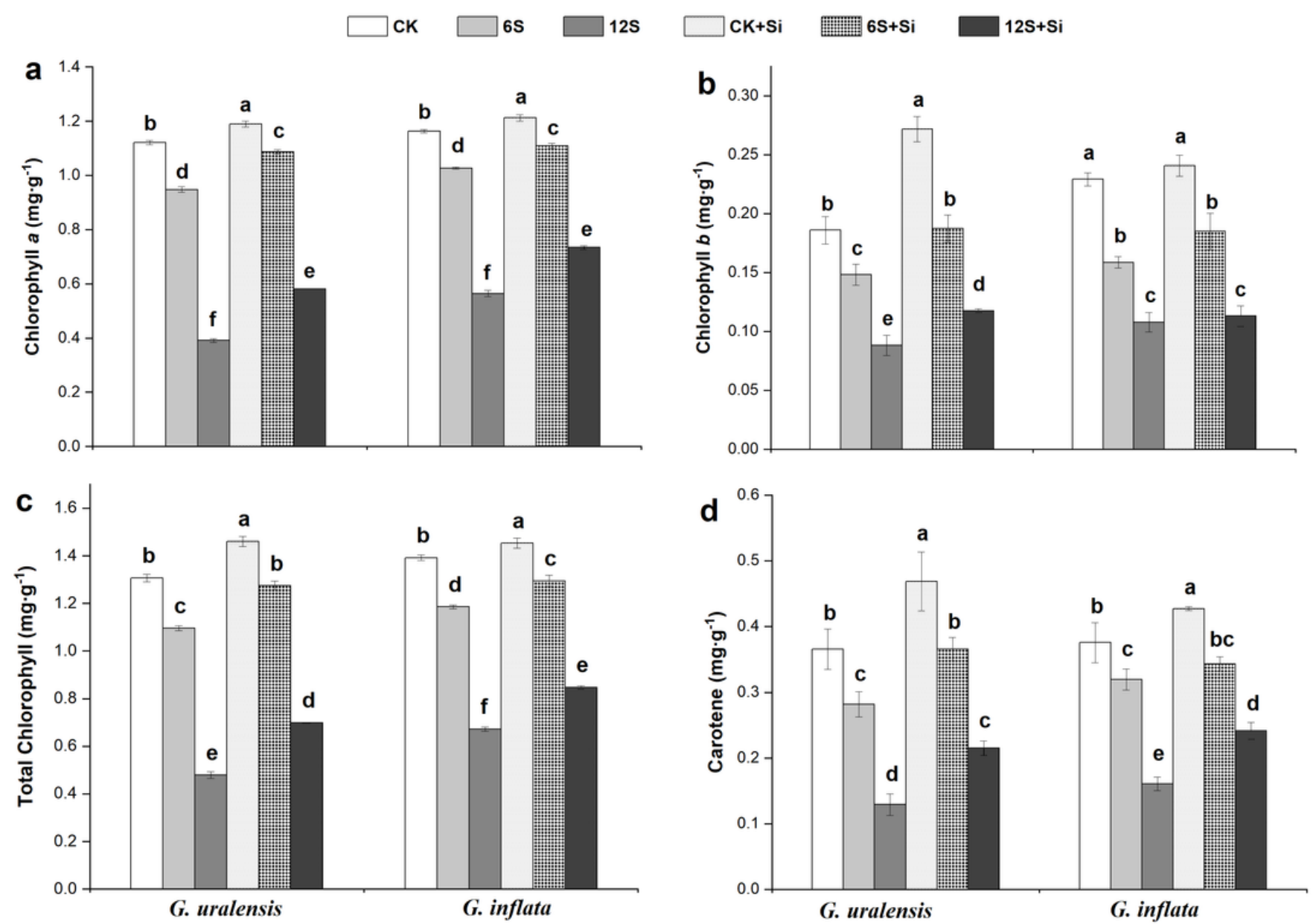

Figure 2

Effects of salinity and silicon on chlorophyll a (a), chlorophyll b (b), total chlorophyll (c), and carotenoid content (d) in the two liquorice species. Data are presented as the mean $\pm S E(n=3)$. Different letters indicate significant differences between the values for one index for the same liquorice species under different treatments $(P<0.05)$ 


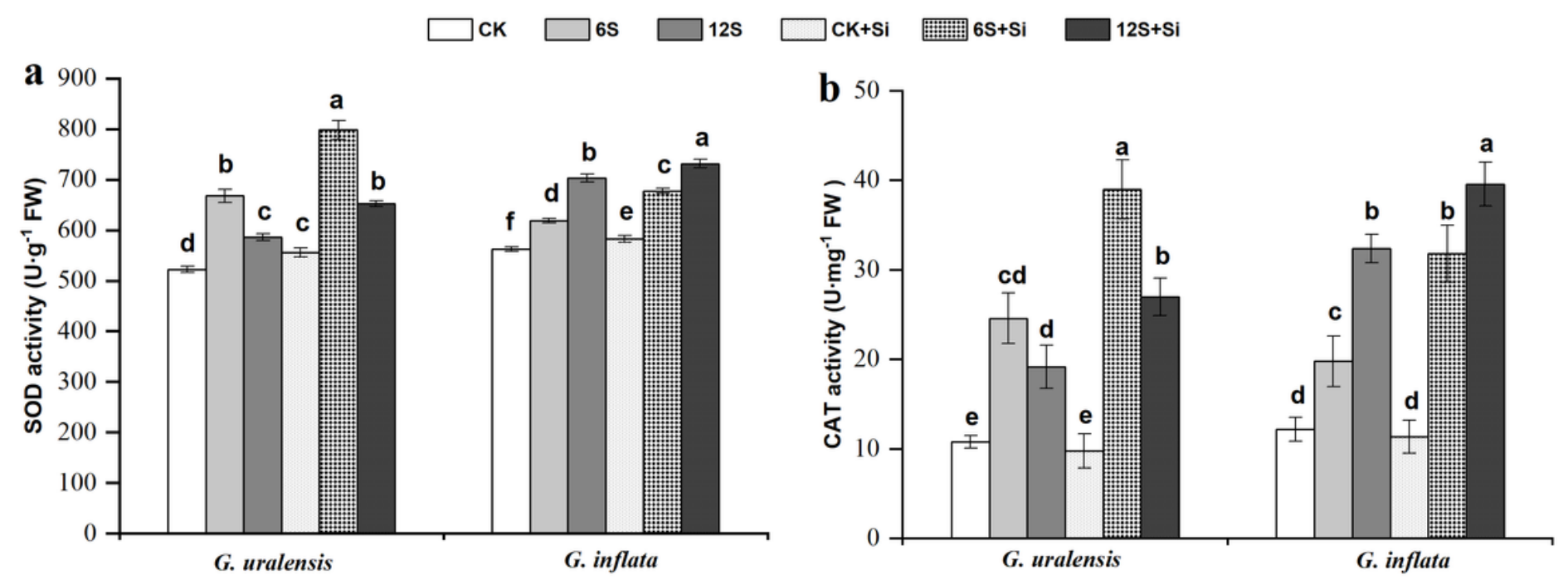

Figure 3

Effects of salinity and silicon on superoxide dismutase activity (SOD, a) and catalase activity (CAT, b) in the two liquorice species. Data are presented as the mean $\pm S E(n=3)$. Different letters indicate significant differences between the values for one index for the same liquorice species under different treatments $(P<0.05)$ 


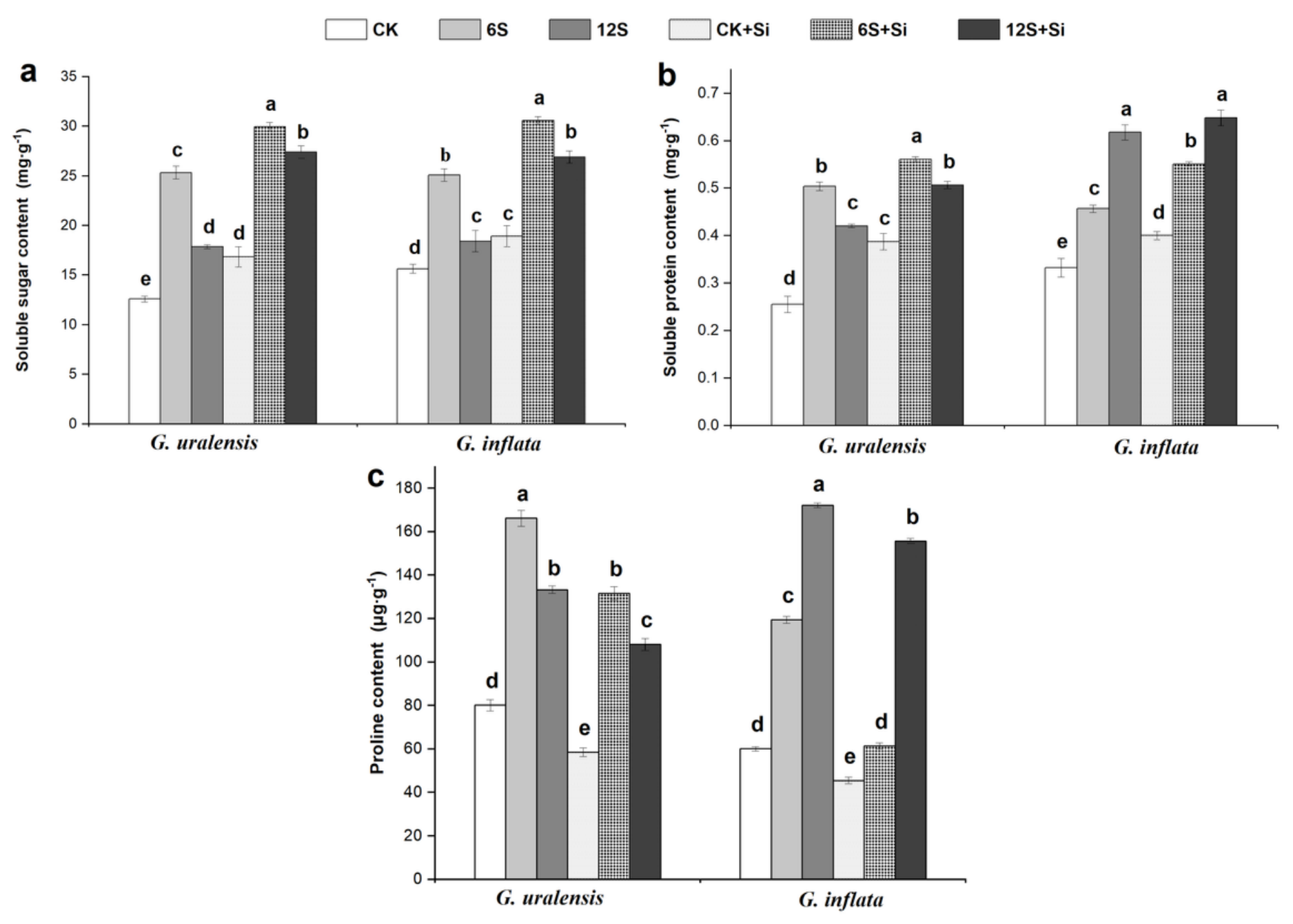

Figure 4

Effects of salinity and silicon on soluble sugar content (a), soluble protein content (b), and proline content (c) in the two liquorice species. Data are presented as the mean $\pm S E(n=3)$. Different letters indicate significant differences between the values for one index for the same liquorice species under different treatments $(P<0.05)$ 


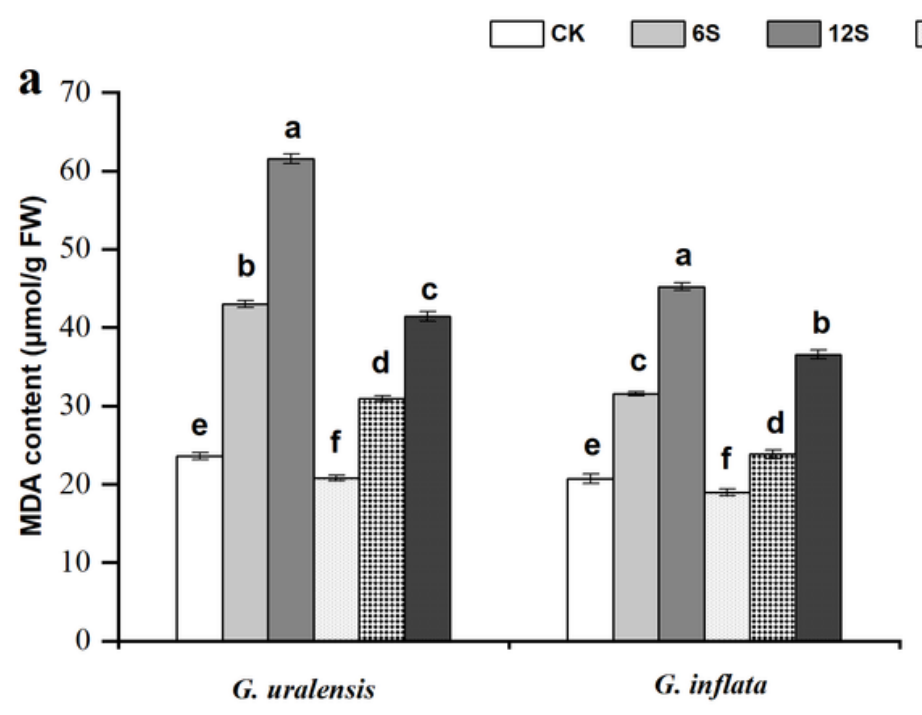

$\mathrm{CK}+\mathrm{Si} \quad$ (m)!\# $6 \mathrm{~S}+\mathrm{Si} \square 12 \mathrm{~S}+\mathrm{Si}$

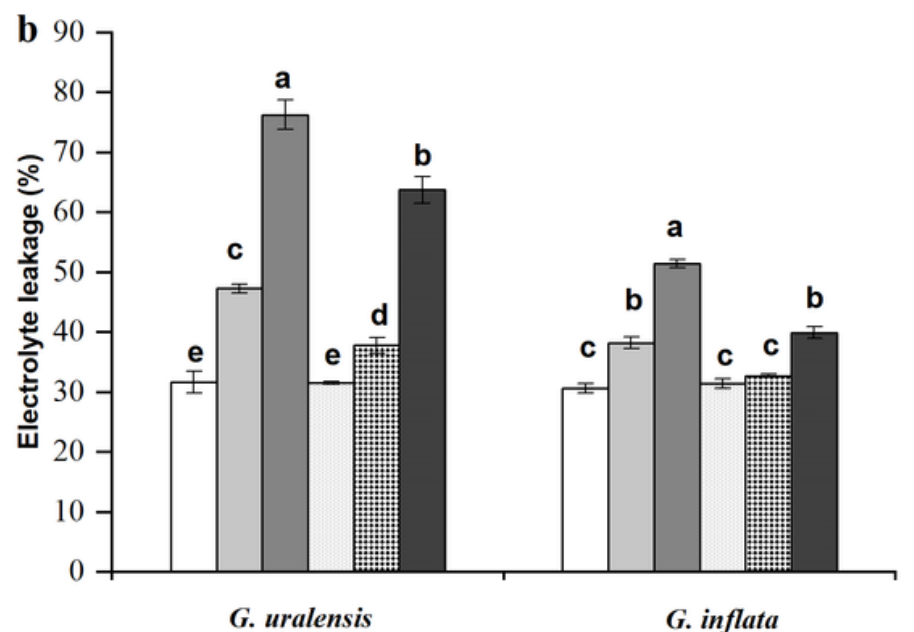

Figure 5

Effects of salinity and silicon on malondialdehyde content (MDA, a) and electrolyte leakage (EL, b) in the two liquorice species. Data are presented as the mean $\pm S E(n=3)$. Different letters indicate significant differences between the values for one index for the same liquorice species under different treatments $(P$ $<0.05)$ 


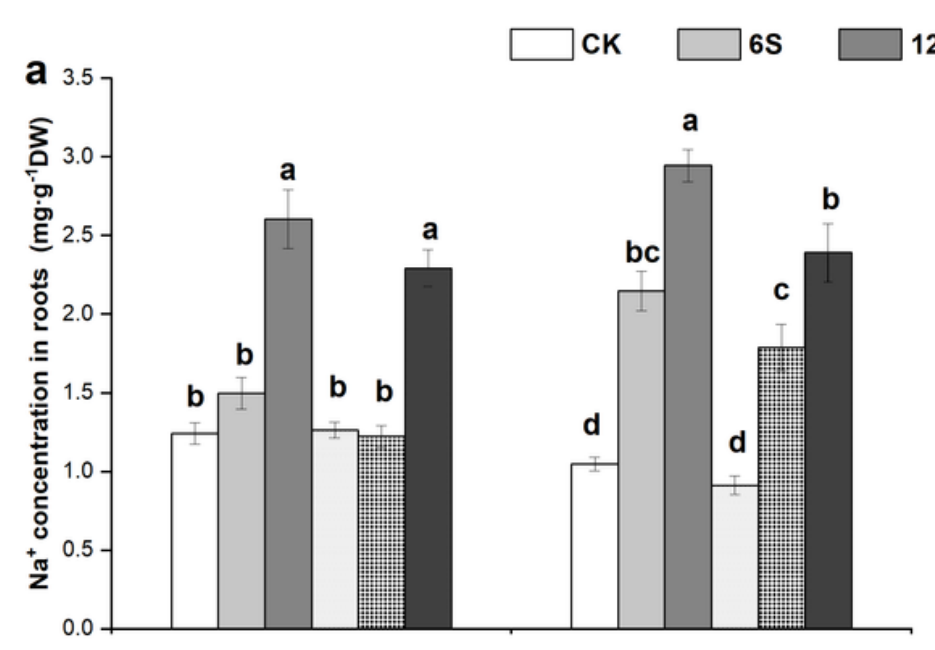

12S
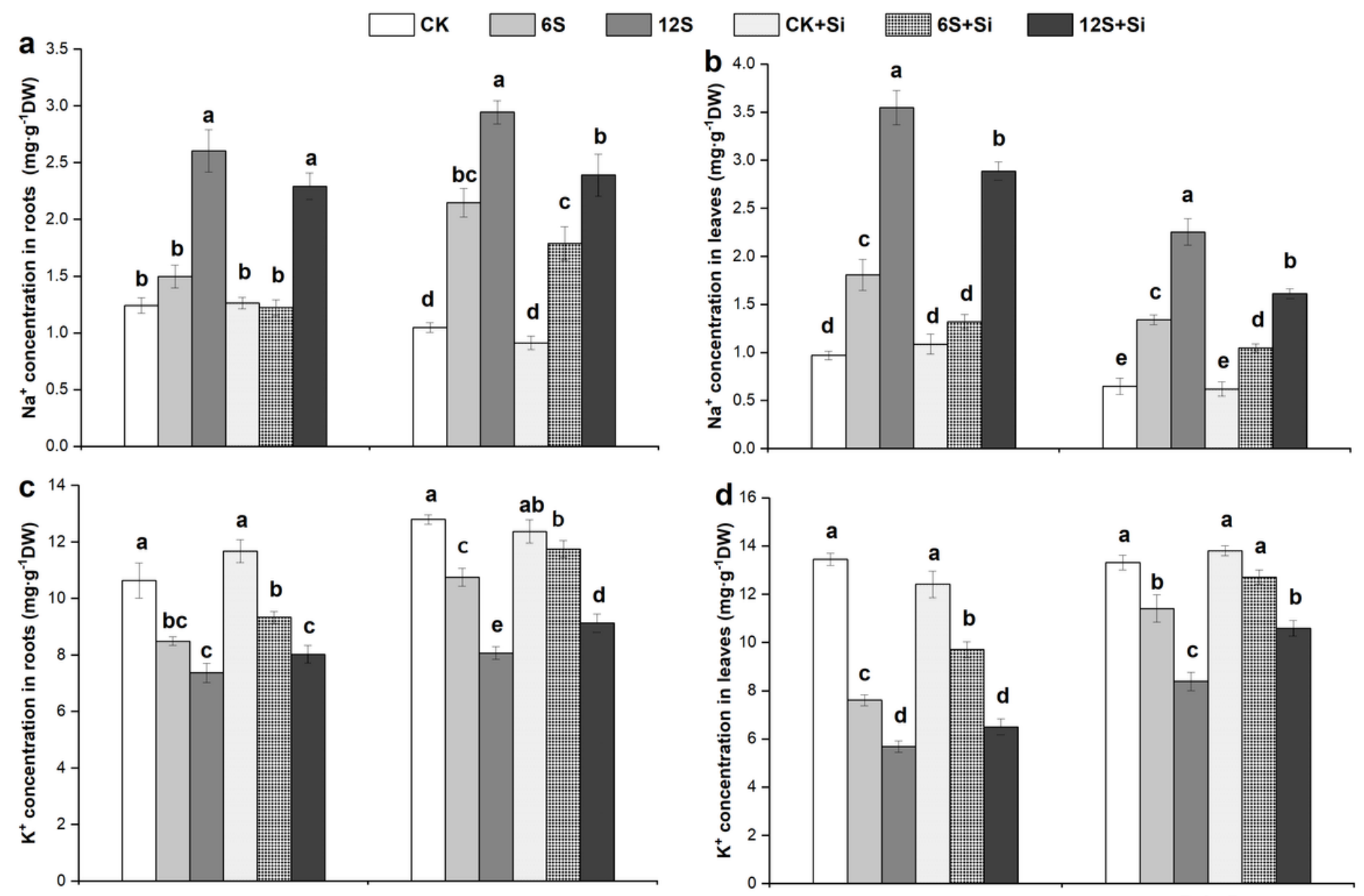

G. uralensis

G. inflata

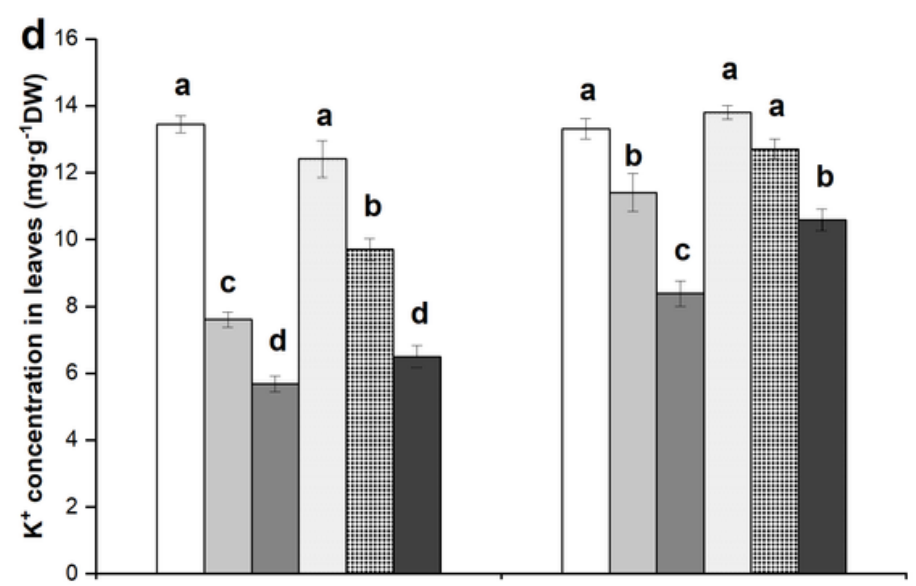

G. uralensis

G. inflata

\section{Figure 6}

Effects of salinity and silicon on Na+ concentration in the root (a) and leaf (b), and K+ concentration in the root (c) and leaf (d) in the two liquorice species. Data are presented as the mean $\pm S E(n=3)$.

Different letters indicate significant differences between the values for one index for the same liquorice species under different treatments $(P<0.05)$ 


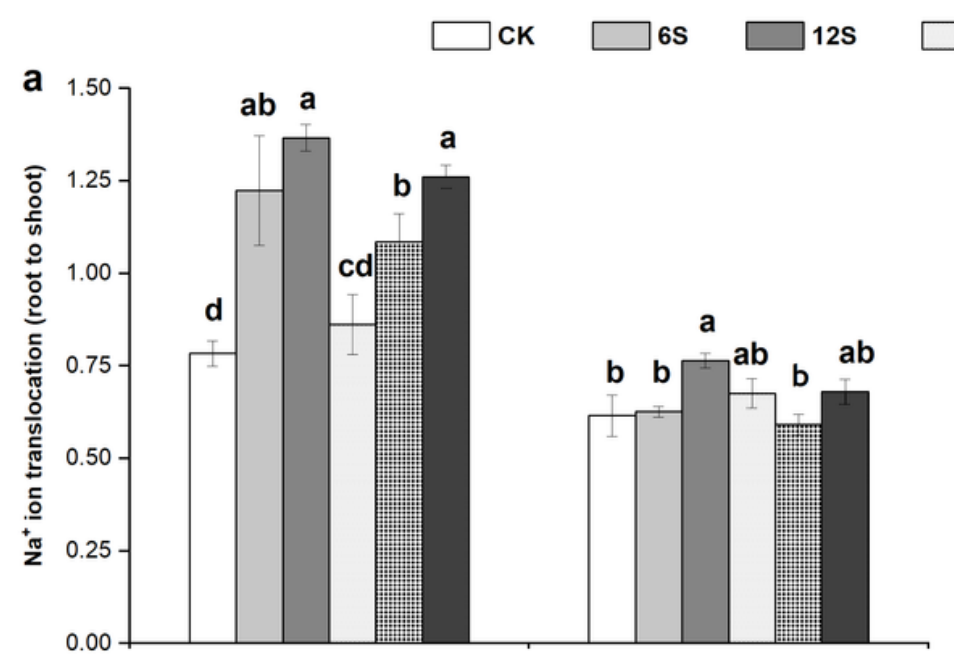

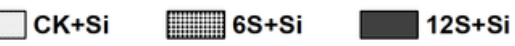

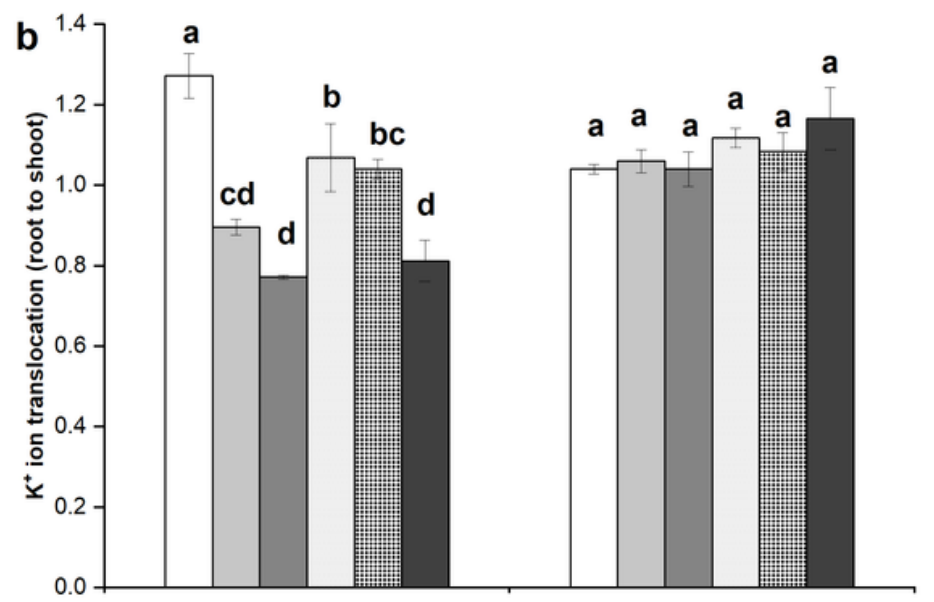

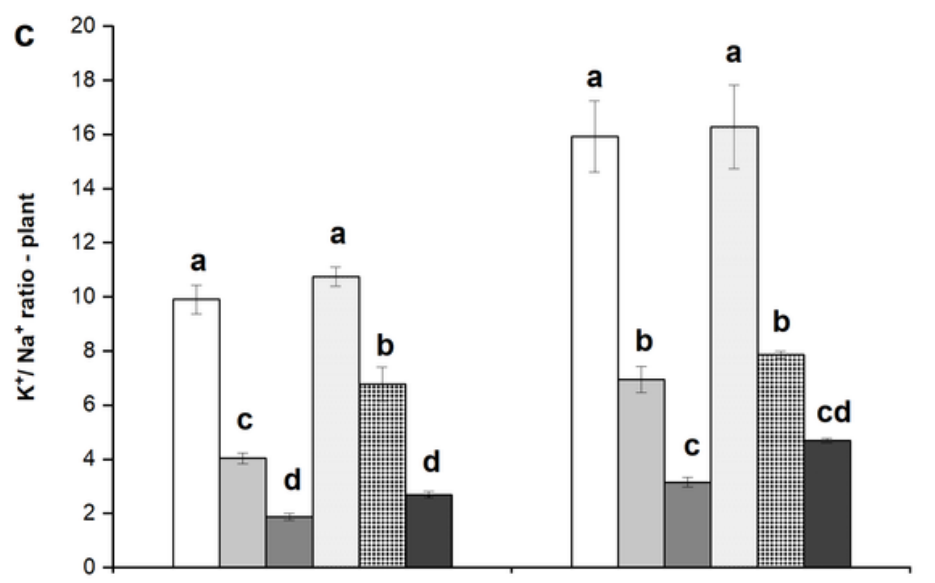

G. uralensis

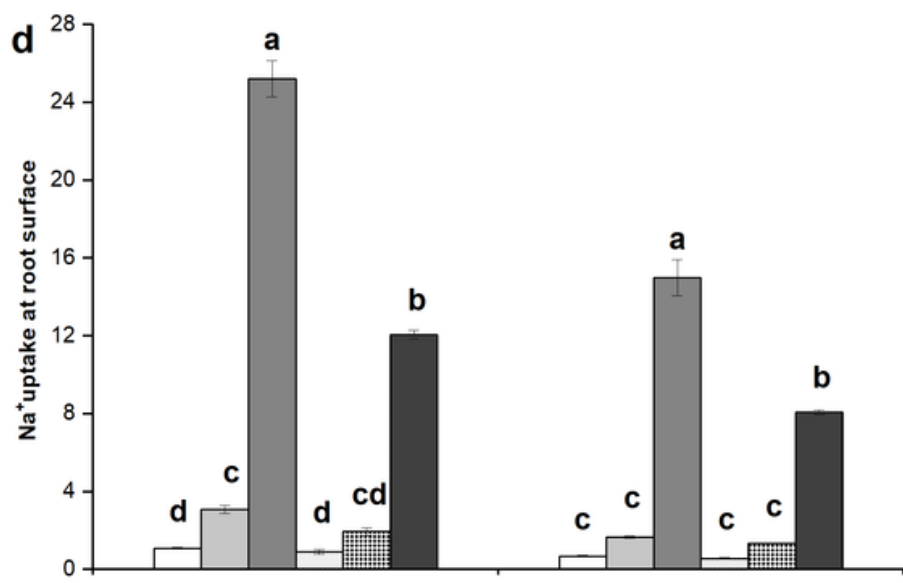

G. uralensis

G. inflata

Figure 7

Effects of salinity and silicon on $\mathrm{Na}+$ translocation (a), $\mathrm{K}+$ translocation (b), $\mathrm{K}+/ \mathrm{Na}+$ ratio (c), and $\mathrm{Na}+$ uptake at root surface (d) in the two liquorice species. Data are presented as the mean $\pm S E(n=3)$. Different letters indicate significant differences between the values for one index for the same liquorice species under different treatments $(P<0.05)$ 